\title{
Coherent THz Transient Spin Currents by Spin Pumping
}

\author{
Lars Bocklage \\ Deutsches Elektronen-Synchrotron DESY, Notkestraße 85, 22607 Hamburg, Germany \\ and The Hamburg Centre for Ultrafast Imaging, Luruper Chaussee 149, 22761 Hamburg, Germany
}

(Received 12 December 2016; published 22 June 2017)

\begin{abstract}
The generation of short spin current pulses is the basis for fast spintronic devices. In thin bilayer systems consisting of a nonmagnetic metal and a ferromagnet, a pure spin current is induced by a precessing magnetization into the nonmagnetic layer by spin pumping. This effect has been experimentally demonstrated at ferromagnetic resonance at $\mathrm{GHz}$ frequencies. Here, it is theoretically shown that transient magnetization dynamics efficiently generates short spin current pulses that exhibit two transient contributions. An effective coherent spin current generation is found far above the ferromagnetic resonance up to $\mathrm{THz}$ frequencies although dynamic magnetization amplitudes are very small in this regime. The results provide a concept for coherent $\mathrm{THz}$ spin current generation.
\end{abstract}

DOI: 10.1103/PhysRevLett.118.257202

In spintronics the electron spin is utilized to build spinbased functional devices. Ultrafast spin currents on a sub-ps time scale are generated by laser excitation in metallic multilayers [1-5] and are predicted for spin injection in semiconductors [6]. These thermally induced spin currents are driven by hot electrons above the Fermi level and are thus limited to conducting materials. The excitation of the electrons result in noncoherent processes, demagnetization effects, and spin-wave excitation. For ultrafast spintronic applications at $\mathrm{THz}$ frequencies, however, a coherent, nonthermal control of spin currents on these time scales is desired.

Spin pumping is another mechanism to inject a pure spin current from a ferromagnet into a nonmagnetic material [7]. Angular momentum is transferred from a thin magnetic film with precessing magnetization into a nonmagnetic material. It covers numerous material combinations of metals, insulators, semiconductors, and topological insulators [8-11] and circumvents the conductance mismatch typically encountered in spin transport. The magnetization is driven at ferromagnetic resonance at $\mathrm{GHz}$ frequencies and induces a pure spin current in the nonmagnetic material. If the spin current is absorbed, it alters the interfacial damping in the magnetic system due to loss of angular momentum [12-15]. In materials with large spin diffusion length, it acts as a spin battery $[16,17]$. The dc component of the spin current is detected in numerous experiments [8,18-24]. Few experiments study spin pumping via burst excitation with burst duration longer than the intrinsic dynamics of the magnetic system [25]. More recently, the ac component has been studied as it yields large spin currents [26-30]. All these experiments are performed at the $\mathrm{GHz}$ ferromagnetic resonance because the spin current is connected to the dynamic magnetic susceptibility [28] and magnetization amplitudes are very small at $\mathrm{THz}$ frequencies [31-33].
In this Letter, transient spin current generation by spin pumping is calculated on the inherent time scale of the magnetic system. It is shown that magnetization dynamics far above the ferromagnetic resonance leads to an efficient spin current generation up to $\mathrm{THz}$ frequencies despite the small precession amplitudes. The formalism of spin pumping [7] is combined with a model of transient magnetization dynamics $[32,34]$ to calculate the spin current transients. Knowledge of their dependence on the excitation of the magnetization is essential for ultrafast spin pumping applications. For $\mathrm{THz}$ pulses a coherent spin current is calculated.

The transient magnetization $\vec{M}(t)=\vec{M}_{0}+\delta \vec{m}(t)$ is described by a static magnetization $\vec{M}_{0}$ and a small timedependent perturbation $\delta \vec{m}$. The spin current $\vec{I}_{s}$ induced by a ferromagnet into a nonmagnetic material is then given by $[7,20,35]$

$$
\vec{I}_{s}=\frac{\hbar}{4 \pi} \frac{g^{\uparrow \downarrow}}{M_{S}^{2}}\left(\vec{M}_{0} \times \frac{d}{d t} \delta \vec{m}+\delta \vec{m} \times \frac{d}{d t} \delta \vec{m}\right),
$$

where $M_{S}$ is the saturation magnetization and $g^{\uparrow \downarrow}$ is the spin mixing conductance. The imaginary part of the spin mixing conductance has been omitted as it vanishes for ballistic and diffusive contacts [7]. For an alternating excitation, the ac spin current arises from the first term of Eq. (1), while the dc spin current arises from the second term.

In the following, a spherical coordinate system is used, where the radial direction is determined by the equilibrium direction of the magnetization $\vec{M}_{0}=\left(M_{S}, 0,0\right)$ and the dynamic part is $\delta \vec{m}=\left(0, \delta m_{\vartheta}, \delta m_{\varphi}\right)$. Then, the first term of Eq. (1) yields the spin current that depends linearly on the magnetization's time derivative:

$$
\vec{I}_{s}^{l}=\frac{\hbar}{4 \pi} \frac{g^{\uparrow \downarrow}}{M_{S}}\left(-\vec{e}_{\vartheta} \frac{d}{d t} \delta m_{\varphi}+\vec{e}_{\varphi} \frac{d}{d t} \delta m_{\vartheta}\right) .
$$




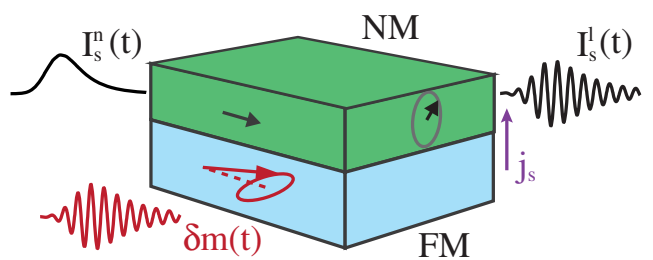

FIG. 1. Scheme of a bilayer system of a ferromagnet (FM) and a non-magnetic metal (NM) typically used in experiments. The transient magnetization is shown in red. The pumped spin current is indicated by $j_{s}$, with a spin polarization given by the spin current vector $\vec{I}_{s}$. The spin polarization of $\vec{I}_{s}^{l}$ oscillates in the plane perpendicular to the equilibrium magnetization. The spin polarization of $\vec{I}_{s}^{n}$ is parallel to the static magnetization. Both exhibit different time dependencies.

The second contribution to the transient spin current originates from the nonlinear second term:

$$
\vec{I}_{s}^{n}=\frac{\hbar}{4 \pi} \frac{g^{\uparrow \downarrow}}{M_{S}^{2}}\left(\delta m_{\vartheta} \frac{d}{d t} \delta m_{\varphi}-\delta m_{\varphi} \frac{d}{d t} \delta m_{\vartheta}\right) \vec{e}_{r}
$$

For a transient magnetization, this term gives rise to a transient spin current, which is spin polarized along the equilibrium magnetization direction. Figure 1 illustrates the bilayer system and the different time dependencies of the spin current components for an in-plane magnetized film. The transient spin current is calculated from the transient magnetization, which is described by a time-dependent response function $\mathcal{R}(t)$. It depends on the temporal evolution of the exciting magnetic field pulse and has been calculated analytically from the Landau-Lifshitz equation to describe magnetization dynamics at $\mathrm{THz}$ frequencies [32,34]. The transient magnetization is related to the field phasor $\vec{h}$ via $\delta \vec{m}(t)=\operatorname{Re}[\mathcal{R}(t) \vec{h}]$. Such THz-induced dynamics that mainly follow Landau-Lifshitz dynamics have been measured in the $3 d$ transition metals $[31,33]$. Recent theoretical work found an additional torque in the Landau-Lifshitz equation that depends on the time derivative of the magnetic field [36]. For frequencies up to $1 \mathrm{THz}$, as considered here, the term is a minor correction.

For harmonic excitation, the spin current can be expressed via the dynamic susceptibility $[37,38]$. Then, the response function is given by $\mathcal{R}(t)=\chi e^{i \Omega t}$, with the excitation frequency $\Omega$ and susceptibility tensor $\boldsymbol{\chi}[34,39]$. Figure 2 shows the spin current components calculated from Eqs. (2) and (3) and $\mathcal{R}(t)$ in a thin bilayer. For the calculations, a permalloy film is assumed $\left(M_{S}=\right.$ $860 \mathrm{kA} / \mathrm{m}, \alpha=0.007$ ) that is magnetized in the film plane in an external field of $\mu_{0} H_{\text {ext }}=5 \mathrm{mT}$. It is excited by an inplane excitation field of $\mu_{0} h_{\varphi}=0.1 \mathrm{mT}$ (see inset of Fig. 2). The resonance frequency is $f_{r} \approx 2.1 \mathrm{GHz}$. The dominant spin current component is the out-of-plane polarized ac spin current that is not detectable by the inverse spin Hall effect in bilayer systems. The in-plane polarized dc and ac spin current components differ by a factor of 5 at resonance. Similar values have been found
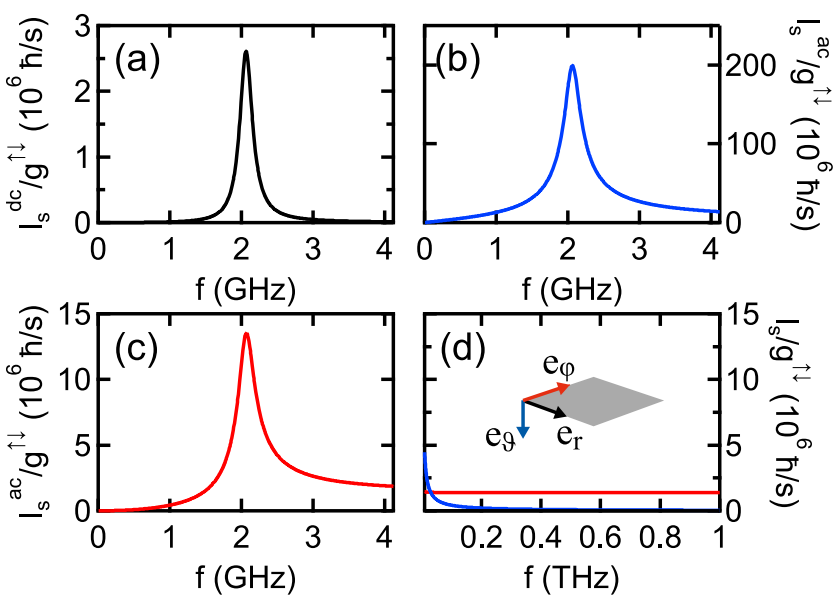

FIG. 2. Spin current in a thin film system for harmonic excitation. The magnetization lies in plane along an external field of $5 \mathrm{mT}$. The excitation field of $0.1 \mathrm{mT}$ in plane and perpendicular to the magnetization. The amplitudes of the spin current components are given. (a) dc spin current polarized along the equilibrium magnetization (black line), (b) ac spin current polarized out of plane (blue line), (c) ac spin current polarized in plane (red line). (d) All components for frequencies up to $\mathrm{THz}$ frequencies. The inset shows the coordinate system with respect to the thin film (gray).

experimentally for inverse spin Hall voltages, while the ratio depends on the actual experimental conditions [28] and on the spin backflow [26]. An interesting feature of the spin current is found for frequencies above resonance [see Fig. 2(d)], where the dc and the out-of-plane spin current drop to zero. The in-plane polarized ac current, however, reduces to an asymptotic value that is only a factor of 10 smaller than at resonance. For a Py/Pt bilayer $\left(g^{\uparrow \downarrow}=\right.$ $3.9 \times 10^{19} \mathrm{~m}^{-2}$ [23]) this off-resonant spin current would be $\vec{I}_{s}^{\mathrm{ac}} \approx 4 \times 10^{25} \hbar /\left(\mathrm{sm}^{2}\right)$. For $\Omega \gg \omega_{r}$, the contribution of the ac spin current is given by [35]

$$
\overrightarrow{I_{s}^{\mathrm{ac}}}=\frac{\hbar}{4 \pi} \gamma^{\prime} \mu_{0} g^{\uparrow \downarrow} \cos (\Omega t)\left(\begin{array}{c}
0 \\
h_{\vartheta}-\alpha h_{\varphi} \\
h_{\varphi}+\alpha h_{\vartheta}
\end{array}\right),
$$

where $h_{\vartheta}$ and $h_{\varphi}$ are the out-of-plane and in-plane excitation fields, respectively, and $\gamma^{\prime}=\gamma /\left(1+\alpha^{2}\right)$, with the gyromagnetic ratio $\gamma$ and the total Gilbert damping parameter $\alpha$ [40]. Far above resonance, the ac spin current does only depend on the excitation field, the spin mixing conductance, and the magnetic damping. In this range, the dynamic magnetization amplitude scales inversely with the excitation frequency [34], but the reduction of amplitude is compensated by the fast temporal change of the magnetization that enters Eq. (1). In the case considered in Fig. 2, the excitation field is given by $h_{\varphi}$ and thus the inplane polarized spin current $I_{s, \varphi}^{\text {ac }}$ is the major component. Note that for a resonant in-plane excitation field the out-ofplane polarized spin current component is largest. 
In contrast, the in-plane polarized component dominates at $\mathrm{THz}$ frequencies. This exchange of the amplitudes is given by the susceptibility of the magnetization. For a thin film, the magnetization precesses on an elliptical trajectory with its semimajor axis in plane at resonance [41]. Far above resonance, the dominant magnetization component oscillates out of plane $[31,32,34]$. This change facilitates the generation of a comparable in-plane polarized spin current at $\mathrm{THz}$ frequencies.

The behavior far above resonance can be used to efficiently generate ultrafast spin currents without the need for magnetic systems with very high resonance frequencies $[42,43]$. The nonlocal behavior of the high-frequency spin current can be obtained from Refs. [14-16,26]. In the limit of long spin-flip relaxation times $\left(\omega>\tau_{\mathrm{SF}}^{-1}\right)$, a noticeable spin accumulation should not be observed above resonance due to the low off-resonant precession angles (see Fig. 2). Its maximum value at $1 \mathrm{THz}$ is estimated to be smaller than $\Delta \mu \leq 2 \mathrm{neV}$, with an angle of $0.1^{\circ}$ [16]. In a good spin sink material $\left(\omega \ll \tau_{\mathrm{SF}}^{-1} \approx 100 \mathrm{THz}\right.$ in $\mathrm{Pt}$ [26]), an ultrafast inverse spin Hall voltage will be generated. The ac inverse spin Hall current is proportional to the spin current and will thus be about ten times smaller as at resonance [compare Figs. 2(c) and 2(d)].

After calculating the remarkable nonvanishing and quite large spin current at high frequencies for a steady-state precession, the transient behavior of the spin current is investigated. A wavelet excitation of the magnetization describes short excitations up to THz. The pulse is written as a product of a Gaussian and a harmonic carrier $\vec{h}(t)=\vec{h} \exp \left[-\left(t-t_{0}\right)^{2} /\left(2 \sigma^{2}\right)-i\left(\Omega t+\phi_{h}\right)\right]$. Here, $\phi_{h}$ is the excitation field phase, $\sigma$ gives the pulse width, and $t_{0}$ is the temporal offset. The transient spin current for short excitations is calculated from the appropriate response function $\mathcal{R}(t)$ [32,35]. Two interesting regimes for the carrier frequency are at resonance and far above it. Both exhibit unique spin current pulse properties.

For transient excitation with a carrier at the resonance frequency $\Omega=\omega_{r}$, the spin current is plotted in Fig. 3. The exciting field pulse is shown in Fig. 3(a) together with the corresponding spin currents $I_{s}^{l}$ and $I_{s}^{n}$. The short resonant excitation leads to a strong spin current. Its amplitude is slightly smaller than the maximum value for steady-state excitation because of the limited duration of the excitation. The resonantly driven magnetization and with it the spin current are delayed relative to the field pulse. $I_{s}^{l}$ oscillates on resonance and is damped out on the time scale of the magnetic damping, as shown in Fig. 3(b). The nonlinear spin current changes on the same time scale. However, it does not exhibit resonant oscillations and its pulse width is compressed compared to $I_{s}^{l}$.

To understand this transient behavior the transient spin current is analyzed in frequency space. The Fourier transformation of the spin current $\hat{I}_{s}(\omega)=\mathcal{F}\left[\vec{I}_{s}(t)\right]$ is [35]

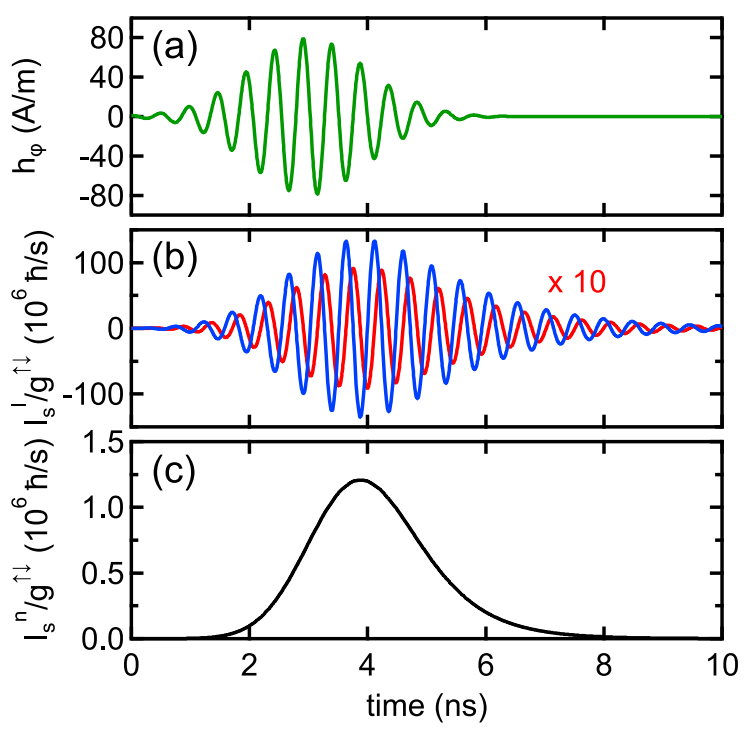

FIG. 3. Transient spin current in a thin film system for resonant wavelet excitation. (a) Excitation field pulse with $\Omega=\omega_{r}$, $\sigma=1 \mathrm{~ns}, h_{\varphi}=0.1 \mathrm{mT}, t_{0}=3 \mathrm{~ns}$, and $\phi_{h}=0$. (b) Out-of-plane (blue line) and in-plane polarized (red line) components of $I_{s}^{l}$. The scale of the in-plane component is magnified by a factor of 10. (c) The spin current $I_{s}^{n}$ is polarized along the static magnetization direction.

$$
\begin{aligned}
\hat{I}_{s}(\omega)= & i \frac{\hbar}{4 \pi} \frac{g^{\uparrow \downarrow}}{M_{S}^{2}}\left\{M_{S} \mathbf{A} \omega \delta \hat{m}+\left[\left(\delta \hat{m}_{\vartheta} \circ \omega \delta \hat{m}_{\varphi}\right)\right.\right. \\
& \left.\left.-\left(\delta \hat{m}_{\varphi} \circ \omega \delta \hat{m}_{\vartheta}\right)\right] \vec{e}_{r}\right\},
\end{aligned}
$$

with $\mathbf{A}=\left(\vec{e}_{r}, \vec{e}_{\varphi},-\vec{e}_{\vartheta}\right)$ and where the $\circ$ denotes convolution. For further analysis, a harmonic time dependence of the magnetization is assumed that is modulated by an arbitrary envelope function $f(t)$. Hereby, any magnetization dynamics is described that follow from harmonic, wavelet, or pulsed magnetic field excitation. It thus covers all dynamics of interest for experiments or technical applications. The magnetization is then given by $\delta m_{\vartheta}(t) \propto \cos (\Omega t) f(t)$ and $\delta m_{\vartheta}(t) \propto \sin (\Omega t) f(t)$, and the Fourier spectrum of the direct spin current results in $\hat{I}_{s}^{l}(\omega) \propto \omega \mathcal{F}[f](\omega \pm \Omega)$, while that of the nonlinear spin current is $\hat{I}_{s}^{n}(\omega) \propto \mathcal{F}\left[f^{2}\right](\omega)$. The latter is solely determined by the Fourier transformation of the squared envelope function of the magnetization. In contrast to the frequency spectra of the magnetization and of the spin current $I_{s}^{l}$ that are distributed around the precession frequency of the magnetization $\Omega$, the spectrum of the nonlinear spin current is shifted around $\omega=0$. The resulting spectrum of $I_{s}^{n}$ corresponds to a transient spin current in the time domain. Steady-state magnetization precession with constant envelope leads to the dc and ac spin currents. Thus, short spin current pulses can be generated depending on the temporal evolution of the magnetization. 


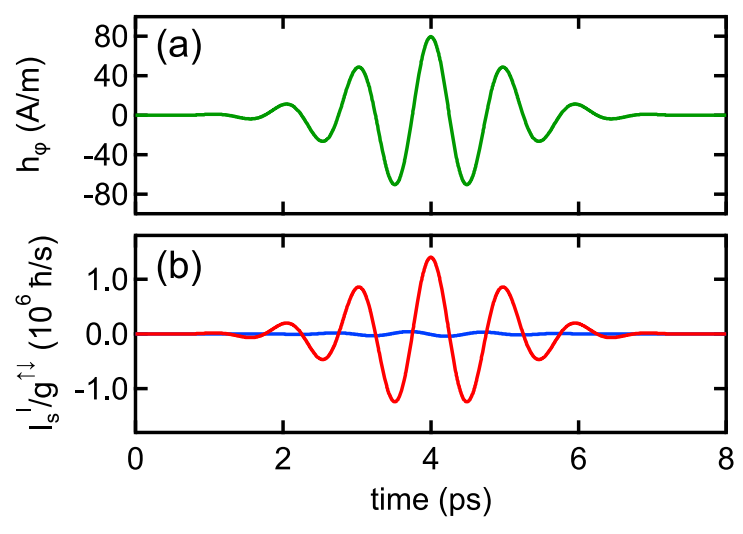

FIG. 4. Coherent spin current generation at $\mathrm{THz}$ frequencies. (a) Excitation field pulse with $\Omega / 2 \pi=1 \mathrm{THz}, \sigma=1 \mathrm{ps}$, $h_{\varphi}=0.1 \mathrm{mT}, t_{0}=4 \mathrm{ps}$. (b) Out-of-plane (blue line) and in-plane polarized (red line) components of $I_{s}^{l}$.

In addition to the generation of spin currents with resonant pulses, an effective spin current generation is possible far above resonance. Magnetization dynamics induced by $\mathrm{THz}$ pulses have been demonstrated to be small in amplitude but to give a coherent response to the THz magnetic field [31-33]. Because of the spin pumping's dependence on the magnetization dynamics, a coherent spin current is excitable on ultrafast time scales. To demonstrate this coherent control, a short linearly polarized THz pulse is applied to the sample, as depicted in Fig. 4(a). The spin current $I_{s}^{l}$ is plotted in Fig. 4(b). The coherent reaction of the spin current to the $\mathrm{THz}$ pulse is clearly visible. As seen before, the in-plane $\mathrm{THz}$ field gives rise to the dominant in-plane polarized spin current, which is comparable in amplitude to the dc or nonlinear spin current at resonant excitation. However, the time scale is 3 orders of magnitude shorter than at resonance. The nonlinear spin current is not shown for the THz pulse as it is in the range of $I_{s}^{n} / g^{\uparrow \downarrow} \leq 0.1 \hbar / \mathrm{s}$.

The excitation fields assumed here are of the same amplitude for the resonant and the THz pump. Magnetic field amplitudes typically used in $\mathrm{THz}$ experiments are several orders of magnitude larger to effectively drive the magnetization. In contrast, $\mathrm{THz}$ spin currents that are comparable to spin currents generated at resonance can be achieved from quite moderate $\mathrm{THz}$ amplitudes. The field intensities of $\mathrm{THz}$ pulses on the order of hundreds of $\mathrm{mT}$ [31] will open the possibility to generate very large coherent spin currents. As the photon energies are small at $\mathrm{THz}$ frequencies, no significant heating and demagnetization are assumed that accompany ultraintense $\mathrm{THz}$ fields [44] or materials with strong spin-lattice scattering [33]. Comparing typical excitation amplitudes of $0.1-1 \mathrm{mT}$ at resonance with field amplitudes over $100 \mathrm{mT}$ for short $\mathrm{THz}$ pulses, the THz spin current will be about up to a factor of 100 larger than at $\mathrm{GHz}$ frequencies. These ultrafast spin currents could be probed by the inverse spin Hall effect or electro-optical sampling [45]. Thus, spin pumping parameters can be investigated on their genuine time scale at $\mathrm{THz}$ frequencies.

In summary, transient spin current injection by spin pumping is calculated up to $\mathrm{THz}$ frequencies providing an off-resonant mechanism to coherently generate $\mathrm{THz}$ spin currents. Remarkably, the ac spin current persists to frequencies far above resonance. Its value is comparable to the dc spin current at resonance. For pulsed excitation of the magnetization, two transient contributions with different time dependencies arise. Coherent $\mathrm{THz}$ spin current pulses can be efficiently generated via spin pumping, which is of practical importance in the field of THz spin dynamics. The mechanism should be applicable to intermetallic junctions as well as to semiconductors and insulators.

I thank Ralf Röhlsberger, Guido Meier, Laura Feiler, and Toru Matsuyama for fruitful discussions. Financial support of the Deutsche Forschungsgemeinschaft via excellence cluster "The Hamburg Centre for Ultrafast ImagingStructure, Dynamics and Control of Matter on the Atomic Scale" is gratefully acknowledged.

*lars.bocklage@desy.de

[1] M. Battiato, K. Carva, and P. M. Oppeneer, Phys. Rev. Lett. 105, 027203 (2010).

[2] A. Melnikov, I. Razdolski, T. O. Wehling, E. T. Papaioannou, V. Roddatis, P. Fumagalli, O. Aktsipetrov, A. I. Lichtenstein, and U. Bovensiepen, Phys. Rev. Lett. 107, 076601 (2011).

[3] D. Rudolf, C. La-O-Vorakiat, M. Battiato, R. Adam, J. Shaw, E. Turgut, P. Maldonado, S. Mathias, P. Grychtol, H. Nembach, T. Silva, M. Aeschlimann, H. Kapteyn, M. Murnane, C. Schneider, and P. Oppeneer, Nat. Commun. 3, 1037 (2012).

[4] T. Kampfrath, M. Battiato, P. Maldonado, G. Eilers, J. Nötzold, S. Mährlein, V. Zbarsky, F. Freimuth, Y. Mokrousov, S. Blügel, M. Wolf, I. Radu, P. Oppeneer, and M. Münzenberg, Nat. Nanotechnol. 8, 256 (2013).

[5] G.-M. Choi, B.-C. Min, K.-J. Lee, and D. Cahill, Nat. Commun. 5, 4334 (2014).

[6] M. Battiato and K. Held, Phys. Rev. Lett. 116, 196601 (2016).

[7] Y. Tserkovnyak, A. Brataas, and G. E. W. Bauer, Phys. Rev. Lett. 88, 117601 (2002).

[8] M. V. Costache, M. Sladkov, S. M. Watts, C. H. van der Wal, and B. J. van Wees, Phys. Rev. Lett. 97, 216603 (2006).

[9] Y. Kajiwara, K. Harii, S. Takahashi, J. Ohe, K. Uchida, M. Mizuguchi, H. Umezawa, H. Kawai, K. Ando, K. Takanashi, S. Maekawa, and E. Saitoh, Nature (London) 464, 262 (2010).

[10] K. Ando, S. Takahashi, J. Ieda, H. Kurebayashi, T. Trypiniotis, C. H. W. Barnes, S. Maekawa, and E. Saitoh, Nat. Mater. 10, 655 (2011).

[11] M. Jamali, J. S. Lee, J. S. Jeong, F. Mahfouzi, Y. Lv, Z. Zhao, B. K. Nikoli, K. A. Mkhoyan, N. Samarth, and J.-P. Wang, Nano Lett. 15, 7126 (2015). 
[12] R. Urban, G. Woltersdorf, and B. Heinrich, Phys. Rev. Lett. 87, 217204 (2001).

[13] S. Mizukami, Y. Ando, and T. Miyazaki, Phys. Rev. B 66, 104413 (2002).

[14] Y. Tserkovnyak, A. Brataas, G. E. W. Bauer, and B. I. Halperin, Rev. Mod. Phys. 77, 1375 (2005).

[15] Y. Tserkovnyak, A. Brataas, and G. E. W. Bauer, Phys. Rev. B 66, 224403 (2002).

[16] A. Brataas, Y. Tserkovnyak, G. E. W. Bauer, and B. I. Halperin, Phys. Rev. B 66, 060404 (2002).

[17] N. Kuhlmann, C. Swoboda, A. Vogel, T. Matsuyama, and G. Meier, Phys. Rev. B 87, 104409 (2013).

[18] X. Wang, G. E. W. Bauer, B. J. van Wees, A. Brataas, and Y. Tserkovnyak, Phys. Rev. Lett. 97, 216602 (2006).

[19] E. Saitoh, M. Ueda, H. Miyajima, and G. Tatara, Appl. Phys. Lett. 88, 182509 (2006).

[20] K. Ando, Y. Kajiwara, S. Takahashi, S. Maekawa, K. Takemoto, M. Takatsu, and E. Saitoh, Phys. Rev. B 78, 014413 (2008).

[21] O. Mosendz, J. E. Pearson, F. Y. Fradin, G. E. W. Bauer, S. D. Bader, and A. Hoffmann, Phys. Rev. Lett. 104, 046601 (2010).

[22] Z. Tang, E. Shikoh, H. Ago, K. Kawahara, Y. Ando, T. Shinjo, and M. Shiraishi, Phys. Rev. B 87, 140401 (2013).

[23] L. Feiler, K. Sentker, M. Brinker, N. Kuhlmann, F.-U. Stein, and G. Meier, Phys. Rev. B 93, 064408 (2016).

[24] Z. Qiu, J. Li, D. Hou, E. Arenholz, A. T. N'Diaye, A. Tan, K. Uchida, K. Sato, S. Okamoto, Y. Tserkovnyak, Z. Q. Qiu, and E. Saitoh, Nat. Commun. 7, 12670 (2016).

[25] D. Sun, K. Van Schooten, M. Kavand, H. Malissa, C. Zhang, M. Groesbeck, C. Boehme, and Z. Valy Vardeny, Nat. Mater. 15, 863 (2016).

[26] H. J. Jiao and G. E. W. Bauer, Phys. Rev. Lett. 110, 217602 (2013).

[27] C. Hahn, G. de Loubens, M. Viret, O. Klein, V. V. Naletov, and J. Ben Youssef, Phys. Rev. Lett. 111, 217204 (2013).

[28] D. Wei, M. Obstbaum, M. Ribow, C. Back, and G. Woltersdorf, Nat. Commun. 5, 3768 (2014).

[29] M. Weiler, J. M. Shaw, H. T. Nembach, and T. J. Silva, Phys. Rev. Lett. 113, 157204 (2014).
[30] J. Li, L. R. Shelford, P. Shafer, A. Tan, J. X. Deng, P. S. Keatley, C. Hwang, E. Arenholz, G. van der Laan, R. J. Hicken, and Z. Q. Qiu, Phys. Rev. Lett. 117, 076602 (2016).

[31] C. Vicario, C. Ruchert, F. Ardana-Lamas, P. M. Derlet, B. Tudu, J. Luning, and C. P. Hauri, Nat. Photonics 7, 720 (2013).

[32] L. Bocklage, Sci. Rep. 6, 22767 (2016).

[33] S. Bonetti, M. C. Hoffmann, M.-J. Sher, Z. Chen, S.-H. Yang, M. G. Samant, S. S. P. Parkin, and H. A. Dürr, Phys. Rev. Lett. 117, 087205 (2016).

[34] L. Bocklage, J. Magn. Magn. Mater. 429, 324 (2017).

[35] See Supplemental Material at http://link.aps.org/ supplemental/10.1103/PhysRevLett.118.257202 for details of the calculations.

[36] R. Mondal, M. Berritta, and P. M. Oppeneer, Phys. Rev. B 94, 144419 (2016).

[37] A. Azevedo, L. H. Vilela-Leão, R. L. Rodríguez-Suárez, A. F. Lacerda Santos, and S. M. Rezende, Phys. Rev. B 83, 144402 (2011).

[38] M. Obstbaum, M. Härtinger, H. G. Bauer, T. Meier, F. Swientek, C. H. Back, and G. Woltersdorf, Phys. Rev. B 89, 060407 (2014).

[39] L. Spinu, I. Dumitru, A. Stancu, and D. Cimpoesu, J. Magn. Magn. Mater. 296, 1 (2006).

[40] Y. Tserkovnyak, H. J. Skadsem, A. Brataas, and G. E. W. Bauer, Phys. Rev. B 74, 144405 (2006).

[41] L. Bocklage, C. Swoboda, K. Schlage, H.-C. Wille, L. Dzemiantsova, S. Bajt, G. Meier, and R. Röhlsberger, Phys. Rev. Lett. 114, 147601 (2015).

[42] R. Cheng, J. Xiao, Q. Niu, and A. Brataas, Phys. Rev. Lett. 113, 057601 (2014).

[43] J. Barker and G. E. W. Bauer, Phys. Rev. Lett. 117, 217201 (2016).

[44] M. Shalaby, C. Vicario, and C. P. Hauri, Appl. Phys. Lett. 108, 182903 (2016)

[45] Z. Jin, A. Tkach, F. Casper, V. Spetter, H. Grimm, A. Thomas, T. Kampfrath, M. Bonn, M. Kläui, and D. Turchinovich, Nat. Phys. 11, 761 (2015). 